\title{
Was the creation of fresenius medical care and davita a step towards a government funded oligopoly to reduce medicare expenditures
}

\section{Introduction}

End Stage Renal Disease impacts the lives of more than seven hundred thousand Americans and their families. ${ }^{1}$ Initially, the CMS funded program, as it was designed in 1972, encouraged nephrologists to open dialysis facilities as a part or extension of their medical practice opening the door to access to care that otherwise seemed impossible. This was a good strategic move in that hospitals were under capacitated and this provided an incentive to provide care giving nephrologists an additional revenue stream beyond seeing and treating patients that otherwise had no options. While the system was being implemented and clinics being both built and providers undergoing an emerging regulatory process in the early 1970's, it was not unheard of for patients to experience outpatient dialysis clinics operating 24 hours a day. Patients were often given whatever time slots for treatment that were available. The government, not being an investor in the process, believed that costs actually would be maintained or come down assuming that immunosuppressive therapy and transplants would be available to lower total expenditures. The problem has been that the government bet that technology would solve the expenditure problem without a concrete strategy to reduce the number of patients entering the system. As a result, the government, unknowingly, actually set up a catchment system in lieu of preventative care that could have both saved patients and maintained a reasonable budget creating a payment structure that combined commercial insurers for a set period of months before Medicare was responsible for reimbursement inciting commercial carriers to buy into the system without any long-term financial commitments. For service providers, for patients under the age of 65 , would bill commercial insurance companies for a specific number of months before Medicare became the primary insurance. This reimbursement structure was the ultimate flaw in the system that led to both consolidation in the industry as well as ballooning expenditures for CMS.

Prior to implementation, the dialysis program was sold to the federal government as a gateway to what universal healthcare may look like in the future. This would be a fully funded government program for a disease that was operating under the radar and could open the discussion of national healthcare system. The problem with this vision was that the condition itself being chronic and extremely expensive to treat. Dialysis, by its very nature, is a poor example of what universal healthcare should look like from multiple perspectives starting with the disease itself. This is a very sick patient population with an expensive and complicated treatment regime. The experiment could have worked if the government got out in front of the disease and established methods for patients suffering from chronic kidney disease to change their daily routines to delay, or in some cases, eliminate the future need for dialysis. Universal healthcare is about balancing costs including those young and the healthy with some requiring treatment creating a pool of individuals that all support each other. As the young and healthy grow older, they are financially supported by new entrants

${ }^{1}$ United States Renal Data System. 2018 Annual Report, 2018. p. 191.
Volume 8 Issue 5 - 2020

\author{
John D Sullivan \\ Ph.D. Boston University, USA
}

Correspondence: John D Sullivan, Ph.D. Boston University, USA, Email jsullva@bu.edu

Received: August 27, 2020 | Published: October 14, 2020

into the pool than can lower the costs through a lighter use of the system creating a balanced structure.

Dialysis is expensive, chronic, and the structure prevents the government from getting ahead of the disease creating a system that is a problematic microcosm of the entire healthcare and Medicare system. A better approach may have been to simply implement a hybrid Medicare plan that extended coverage to those that otherwise couldn't afford access to chronic kidney disease combined with incentives to encourage patients to seek care and treatment prior to complete kidney failure. Under this scenario, certain provisions in the bill may have needed to be included providing protections for patients that preferred commercial coverage leading to restrictions and penalties on employers that would have simply pushed employees onto the government system. Additionally, given the tremendous cost of treating end stage renal disease, Medicare's revenue would also have needed to be increased raising funds through an increase in CMS tax to cover these patients.

Unfortunately, the goals of the government never materialized and created a market that encouraged dialysis centers into consolidation over time to reduce expenses through leveraging third-party payers. Dialysis, at one point, had been highly fragmented but was prime for consolidation for those that had the vision to see huge profitability through mergers and was on the radar of private equity firms that saw the opportunity to bring these fragmented dialysis pieces into a larger firm with leveraged assets and a market that was poised to grow as the incidence and prevalence of diabetes and hypertension became more common. The initial budget for the program was estimated at approximately $\$ 100$ million with a realistic view of maybe $\$ 250$ million on the high side in its first year of operation. By 1979 , expenditures for dialysis were over $\$ 1$ billion. ${ }^{2}$ The program was supposed to be an insurance system to provide access to care, but turned out to be a way for third party commercial payers to supplement their costs through a limited coverage time by using the government so long as the service providers could operate below the radar in so far as a weighted average revenue stream supplemented by private insurance companies.

${ }^{2}$ Eggers P. Medicare's End Stage Renal Disease Program. Health Care Finance Review. 2000;22(1):55-60 
Unfortunately, because the program was set up as a catching mechanism for patients that didn't have coverage or access to the limited care that would ultimately lead to fatality, there were no preventative care solutions within the bill so Medicare would simply be the coverage provider after a waiting period that has been slowly extended over the decades. Due to this simple flaw in the bill, which was maybe understandable given what was presented to Congress, any commercial insurance carriers that bridged the gap between diagnosis and Medicare had no real financial incentive for preventive care for chronic kidney disease (CKD) knowing that patients would ultimately be the sole financial responsibility of the government. From the government's perspective, given the first cost projections, this may have made sense. If technology was to assist patients going forward that would reduce expenditures with the assumption that diabetes and hypertension would not take a foothold in society, the formula works. Transplantation and technology were the government's "ace in the hole." Except, there was a significant change in one of the variables. First, technology was slow to develop and second, there were far more patients than expected in the pipeline and would continue as the rate of diabetes and hypertension continued to rise.

Despite all the advancements and growth, after almost 50 years, this policy hasn't really changed, but merely been extended the time period whereby Medicare would cover patients financially. In addition, the government has attempted to contain expenditures through a "per treatment" system that may have significant side effects for access to care that may or may not have been intentional without the ability to assess what the true problem is. Frankly, the government is still acting as a catchment system for patients diagnosed with the disease attempting to reduce the cost outlays through per treatment adjustments in lieu of keeping patients off of dialysis. This logic is counterintuitive if the goal is to lower total the budget and actually increase patients' quality of life by preventing or reducing the numbers of potential patients that either have chronic kidney disease (CKD) and thus lowering the number of Americans that require the treatment.

\section{The evolution}

The initial system worked in that it covered patients that otherwise may have been subjected to either lack of access to dialysis or in extreme cases, committees to evaluate whether or not they be allowed to a limited amount of care via dialysis. The technology available at the time was somewhat crude by today's standards, but effective in sustaining the lives of patients suffering from ESRD that otherwise would have been subjected to these committees. The treatment was clinically revolutionary but created a significant problem in access to expensive equipment and clinicians leading to traditional support via insurance carriers as being somewhat reluctant to coverage given the large reimbursement required for each dialysis payment. With the support of the federal government, clinics opened quickly and patients almost instantly had access to care although capacity needed to be expanded with an underlying infrastructure secured from government reimbursement support. As access grew, so did buy in from third-party commercial insurance payors that knew the federal government would be covering the bulk of the cost of care and their financial liability was limited. Commercial insurance carriers, based on a weighted average, would become the ultimate revenue source for service providers, from a profitability standpoint, for the dialysis industry and continues to do so as the government attempts to reduce their costs with the variance in reimbursement being significant between traditional insurance and Medicare payments. That said, insurance companies covering those under the age of 65 knew that the future expenses associated with patients for this disease would be covered under the federal government. At worst, these entities understood that any future liability would be limited with patients not surviving to the Medicare as secondary payer designation or living to transition to CMS being the primary insurance provider.

Nephrologists, that had merely practiced as an extension of urology, treating patients with a chronic disease that had ultimately led to fatal conclusion, had transitioned into millionaire entrepreneurs with more patients than their practice had dialysis machines could care for. As a result of the surge in patients, practices increased with recruitments of new nephrologists over the first decade of Medicare coverage as the need demanded. Many of these physicians became partners and experienced the wealth produced by the expanding of outpatient clinics as demanded by the patient population. This was a benefit of the time, but not sustainable as the capacity rose up to meet the demand of those requiring dialysis. The expansion, from a patient perspective, was truly beneficial because patients were no longer required to have dialysis at a clinic that operated 24 hours a day and had better treatment time options. Profitability, which is debatable if would include the cost of overtime, may have been reduced over this time as clinics opened and patients had more options, but did create an economy of scale with a physician group that owned more than one clinic in a specified geographical space. Patients, especially those that may have other health conditions related or not related to ESRD, had options to better suit their treatment needs. The system seemed to be working, but there was an underlying potential problem. New physicians could not afford to by an equity stake in the dialysis clinic and while they enjoyed the medical director fees the value of these clinics had become clear. To make matters more difficult, these younger nephrologists that entered these practices that were earning a good salary, but their hands tied through covenants not to compete meaning they were prohibited from opening their own clinics even if they had the capital.

With acquisitions of these independent clinics, new nephrologists' options were even more limited. As consolidation began to occur with the original nephrologists easing into retirement, more new nephrologists were needed to serve as medical directors that came with tight non-compete agreements and were legally attached to the acquiring entity. This action by the acquiring company prevented competition and the syphoning of patients into to new clinics that would have created a more economic level playing field. From the government's perspective, this may have been a tactic to reduce competition in the market adding to more pressure against thirdparty insurance payors and thus reducing expenditures for CMS. It also provided a potential joint venture equity stake for nephrologists that wanted a smaller minority equity stake. However, with a smaller stock ownership stake, the exit options were limited to the primary shareholder such as Fresenius, DaVita, U.S. Renal Care, ARA etc. This was an olive branch to incentivize the nephrologist to keep patients in a clinic with, ironically, no real financial incentive on the value of equity in the event of a clinic sale to the provider since the value had been established the moment of the physician's "equity buy in." The clinic can certainly rise in value, but has a limit given capacity and the significant investment by the dialysis chain limits what can be earned on the back-side.

In a sense, because the shift has moved from the nephrologist controlled clinic to the for-profit publicly traded outpatient unit, 
many of the benefits to the physician and the direction of the practice has been moved over to corporations with their benefit with little or no monetary compensation to the actual providers of care to these patients. In a sense, nephrologists have become employees of the large dialysis chain. While treatment modalities are still in the hands of these physicians, corporations through medical director fees, can have a significant influence on prescriptions moving towards more profitable modalities such as in-center dialysis that utilizes fixed costs and reduced variable costs (dialyzers and lines) to leverage profitability for a clinic verses, what would seem to be logical, at home dialysis that has no leverage in fixed costs, but a significantly a heavier variable cost component with more dialysis utilization.

\section{Treatment options}

Treatment options for dialysis have not expanded over the decades with prescriptions for modalities outside in-center hemodialysis being stagnant. Both peritoneal dialysis and home hemodialysis have lagged significantly behind the traditional in-center hemodialysis option despite significant advancements in technology. ${ }^{3}$ Optimists show that while these modalities have lagged behind prescriptions for dialysis, they have grown significantly, although they still remain significantly behind hemodialysis. ${ }^{4}$ Both treatments are far behind the preferred in-center dialysis modality, but it remains unclear as to why. Some research suggests that it is a profitability strategy to promote outpatient dialysis over other treatment modalities. ${ }^{5}$ Unfortunately, for those without a transplant, End Stage Renal Disease really only have three options that include the traditional in-center hemodialysis treatment, outpatient peritoneal dialysis, and home hemodialysis. Some have blamed it on a lack of training and others on the dialysis providers, but options have been limited over the years leading to a tremendous number of patients relying on in-center hemodialysis for their only treatment option. ${ }^{6}$ Given the current state of the dialysis provider market, others have argued that profit has driven patients into the clinics and away from potential other treatment modalities. ${ }^{7}$ From a clinical perspective, this can be rather troubling given that peritoneal dialysis and home hemodialysis may have better treatment outcomes or at least the same outcomes as in-center hemodialysis although the setting of PD and Home Hemo provides better quality of life. ${ }^{8}$ If this is the case the question becomes whether or not in center hemo dialysis is being driven by the nephrologist, that may still have an economic or equity interest in a clinic, or the dialysis provider which is subject to their respective shareholders. It is a relatively easy equation to see the profit motives of an in-center unit running at 3 shifts per day would leverage the fixed assets making it more profitable than PD or home therapies. It happens to be a somewhat financial argument however, for a nephrologist, even if not a joint venture participant, through medical director fees and non-compete agreements, to sign on to a treatment modality that benefits their respective partner.

\footnotetext{
${ }^{3}$ United States Renal Data System. Annual Report. 2018. P. 317

${ }^{4}$ www.healio.com/nephrology/practice-management/news/online/\%... dialysis-providers-in-2017 Accessed 3/6/2020

${ }^{5}$ Sullivan, John D. The State of the Treatment of End Stage Renal Disease in 2019 in the United States. Journal of Nephrology \& Urology. 2018;5(1):036. ${ }^{6}$ Sullivan, John D, Stern. The State of Kidney Failure in the United States in 2018. Journal of Urology \& Nephrology. 2018;5(1).

${ }^{7}$ Sullivan, John D. The State of the Treatment of End Stage Renal Disease in 2019 in the United States. Journal of Nephrology \& Urology. 2018;5 (1):036. ${ }^{8}$ Ishani A, Slinin Y. Comparative Effectiveness of Home-Based Kidney Dialysis Verses in Center or Other Outpatient Kidney Dialysis Locations - a systematic review." Queri. Department of Veterans Affairs: Heath Services Research \& Development. 2015
}

Among the most influential top-ten providers, treatment modalities outside the traditional in-center hemodialysis are rather non-measurable. Home hemodialysis is still so insignificant the United States Renal Data System simply reports them as a home therapy grouped with peritoneal dialysis. Reports of the top ten providers reflect a 7.3\% compounded annual growth of HHD from 2013 to 2019 , but still reflects a $1.5 \%$ treatment modality (2017) despite the significant advances in the simplicity of the NxStage HHD system. Peritoneal dialysis has done a bit better capturing $9.6 \%$ of the treatment modalities in 2017. ${ }^{9}$ This is in line with past percentages of treatment modalities for peritoneal dialysis as a percentage of patients being treated outside of transplantation, which is more concerning. Actual numbers for peritoneal dialysis and home hemodialysis show better growth if measured individually, but still do not have an impact on the traditional in-center hemodialysis modality. Peritoneal dialysis, in 2019 , had roughly 50,400 patients up from 39,800 patients in 2015 reflecting a growth rate of $6.1 \%$. Home hemodialysis during the same period served 8,807 patients in 2019 among the top ten dialysis providers growing from 6,558 or $7.65 \%$ on a compounded growth rate from 2015. ${ }^{10}$ While this is an exceptional growth rate for these two treatment modalities given the mortality net increase of approximately $3 \%$ for those requiring dialysis, it still lacks significantly behind the majority treatment modalities on a total basis looking at treatment options.

This is not to say that these treatment modalities are superior to the traditional to in-center hemodialysis. For example, patients that are prescribed peritoneal dialysis do run the risk of developing peritonitis. This condition should not be discounted as a simple side effect as hospitals are havens for both bacterial and viral infections that could impact a patient's success undergoing this treatment. Studies have suggested that some countries are better at reducing these rates than others although the data seems to suggest that tendencies for patients to have this condition tend to be relatively narrow. ${ }^{11}$ This may be a reflection of a physician's selection of the best candidates for this treatment other than the outcomes of a patient that may not have been the best candidate for this type of modality. Obviously, the ideal treatment modality, or even better preventing dialysis, is transplant and this has also grown over the years however with the lack of organs, both live and cadaver, have limited this as an option for patients. Although there are some bright signs with this treatment. In 2009, there were 16,829 kidney transplants in the United States. By 2019 , transplants had increased to 23,401 representing a compounded growth rate of $3.4 \% .^{12}$ The growth rate is encouraging, but not enough to reduce the number of patients requiring dialysis by keeping pace with ESRD increases each year net of mortality. As a result, peritoneal and HHD must be utilized more to both contribute to patient's quality of life as well as an overall economic benefit to the government.

As a treatment, it is important to note that transplantation is nothing more than that. It is not a cure, nor should it be sold to patients as such. This is just another treatment option that happens to be very favorable to patients. The key to this treatment, like all others, is to prevent patients even if the availability of organs continues to improve. This should not be thought of as a catchment for patients that are on the verge of dialysis but rather the nephrologists and insurance

${ }^{9}$ www.healio.com/nephrology/practice-management/news/online/\%... dialysis-providers-in-2017 Accessed 3/6/2020

${ }^{10}$ Growth in Home Dialysis. Nephrology News and Issues. 2020. p. 26

${ }^{11}$ Peritonitis rates vary by country, facility. Nephrology News and Issues. 2020. p. 16

${ }^{12} \mathrm{https}$ //optn.transplant.hrsa.gov/data/view-data-reports/national-data/\# 2020 
payers should be looking for ways to keep payers off of dialysis. Only by doing so will ultimately bridge the gap between those requiring a kidney and those that have availability to an organ can close. Otherwise, it seems as though the availability of kidneys ready for transplantation will never catch up to the number of patients making the transition to dialysis.

\section{The economics}

Dialysis is an extremely expensive method for caring for patients regardless of the treatment modality. The bulk of the cost is tied to the treatment itself, but also hospitalizations as a result of the disease combined with physician visits. The problem is that this group of patients is a health compromised group requiring a balance of care whereby dialysis is the result of other issues generated by either diabetes or hypertension. Patient compliance can also be a factor in hospitalization rates and keeping the costs down overall. From a patient standpoint, the restrictions can be challenging especially when it comes to diet and liquid consumption. While some nephrologists may describe fluid consumption as "they want what they cannot have," the restrictions are difficult to comply with even with fully functioning kidneys and attempting to live the life as a dialysis patient. That said, the disease is expensive to treat and the patient population, net of mortality, will continue to grow at approximately $3 \%$ per year. ${ }^{13}$ Some of the predictions of forty years ago have started to become a reality as transplants over the past few years have increased significantly. However, while the amount of kidney transplants has increased from 16,900 in 2009 to 23,401 in 2019 representing a $4.2 \%$ compounded annual growth rate. ${ }^{14}$ This is a positive trend given that transplants have lacked behind the patients' needs for years. However, even with a one percentage growth over the population that requires a kidney, the population won't simply get to where they need to be or what the government was promised for decades.

The economics of dialysis is relatively simple in that the patient population is relatively non-compliant (understandably) coupled with a direct treatment cost that runs under a hundred thousand dollars coupled with hospitalizations that can push that number significantly higher. Expenditures for dialysis, as judged by the United Renal Data System, on a Medicare Basis, would top $\$ 33.9$ billion or $7.1 \%$ of the CMS budget for a total patient population that is less than $1 \%$ of the patient population. ${ }^{15}$ Previous years the federal government paid $\$ 26.2$ and 26.7 for dialysis. ${ }^{16}$ Given the high expenditures, this should be on the radar of not only CMS, but also the federal government. It is understandable that there is always a crisis in Washington. The financial meltdown in 2008 and recently the corona virus that will lead to yet another recession. One would argue that it is not at the peaks of an economy is when you make changes, but when times demand it. If the government is in need of cash and has been looking the other way for fifty years, now is the opportunity to put in place a system that would ensure that patients are being taken care of and future expenditures are under control.

The problem is one of the pitchers or the catcher. The United States Government has always been the catcher. If one is willing to provide unlimited care for a high cost group of patients regardless of age, it

${ }^{13}$ United States Renal Data System. Annual Report. 2018. p. 317

${ }^{14} \mathrm{https}$ //optn.transplant.hrsa.gov/data/view-data-reports/national-data/\# Accessed March 19, 2020

${ }^{15}$ USRDS Annual Report 2016, Chapter 9

${ }^{16}$ USRDS Annual Report 2016, Chapter 9 would be logical to jump out in front of those patients and delay the progression of ESRD as long as possible. From a government coverage perspective, this hasn't happened. The government has served as the coverage for patients that have gone through their secondary payor period and been the support thereafter. They haven't been out front fighting to prevent this disease from progressing. The disease run will continue to grow and it extraordinarily expensive to treat. No one seems to be in front of the problem. Expenditures can be significantly reduced if the government can get out in front of the problem. Third party payors likely look at this as a three-year problem that can be shifted off to the government so just look at it as an "accounting write off." Essentially, there is no incentive for even the private sector to push for preventative care.

The simple approach would dictate that one would drive revenue down and reduce expenditures through a thoughtful strategy that would both benefit the patients as well as the nephrologists. As for an organization that is a recipient for patients, this makes sense in that they are under the responsibility to cover a sick population without the ability to control expenditures from the from side of the disease. The incentive is to reduce costs through creative means such as capitation and patient outcomes. From a financial perspective, this is a disaster. It creates costs on the provider side, patients that are non-compliant may be dumped on other systems, and providers margins are reduced. If CMS truly wants to reduce expenditures, they need to get out in-front of the disease and prevent it from happening. It is far less expensive to treat the disease before renal failure has set in. From an economic standpoint, aside from the patient, the economic impact can be far greater than the patient if one considers the likelihood that the patient may drop out of the workforce and end up on disability support that provides both an economic drain and a lower contribution to the general economic community than one not using government support. Unfortunately, this is not a problem that is going away anytime soon. Renal failure is a problem that will continue for the foreseeable future as the incidence and prevalence of diabetes and hypertension continue to grow. Now seems the time that CMS, if the goal is to reduce expenditures and improve patents' health, needs to be a bit on the proactive side.

\section{The oligopoly}

End Stage Renal Disease, as an industry, is, by its very nature, was a fragmented market and ripe for consolidation. By current accounts, it is no longer a fragmented market because there are realistically no acquisition targets left to purchase. The market changed dramatically from the 1990s to current day. Nephrologists of the first generation sold their respective businesses for a nice profit, mostly based on price per patient until the late 1990s, until there were no real independent controlled dialysis centers. What the market is left with is a group of highly concentrated dialysis operation companies that at the top control the entire market. Fresenius Medical Care and DaVita own approximately $85 \%$ of the top ten providers with the next eight providers only having a $15 \%$ market share ${ }^{17}$ Even independents struggle to keep their head above water given their lack of market influence and leverage with third party commercial carriers. Without geographical leverage, these smaller providers could ultimately be undercut by larger providers such as Fresenius and DaVita and economically forced out of the market due to a lack of negotiation power with third party commercial insurance carriers.

${ }^{17} \mathrm{https} / /$ www.healio.com/news/nephrology/20180227/the-largest-dialysisproviders-in-2017-more-jump-o Accessed 3/6/2020 


\section{Conclusion}

There is no doubt that the care of these patients is a critical component of the American healthcare system. Patients would otherwise be subjected to restricted care resulting in a higher mortality rates having a devastating impact on the general economy and families given that more of the burden would be placed on the commercial sector with a understandably financial pushback given the longer wait if not the elimination of government coverage not to mention patients that may not have access to insurance to begin treatment. The problem is that the federal government continues to look at the problem from the back end and not from a preventative measure. The best way to combat this disease is to prevent it from happening by taking measures to have patients diagnosed early with diabetes, hypertension, and glomerulonephritis, and taking the step to ensure that these patients have continual care to treat these conditions to eliminate or delay ESRD from occurring. This is the ultimate and optimistic cure for the problem, but the market is built for something else. End Stage Renal Disease is an industry controlled by two companies and this is reflective of a government that is only focused on expenditures and not prevention. Does DaVita and Fresenius Medical Care really care about prevention of ESRD? In the oligopolies defense, they are merely the recipients of the patients from a system set up to prescribe dialysis. Medicare walks a thin line reimbursing as little as possible knowing that commercial third-party payers will make up the difference and provide profits for these publicly traded giants.

Nephrologist's incentives to reduce dialysis is also a bit skewed towards treatment rather than prevention given their respective roles as medical directors of clinics their group oversees. It is also understandable to see how these physicians are more comfortable placing patients on dialysis under the controlled guidelines of an outpatient clinical staff. This not to say that nephrologists are acting with unethical or monetary intentions, but rather it is somewhat with ease to shuffle patients along to the clinic and more difficult to manage a patient's training and ability to maintain their own care via peritoneal or, even worse, home hemodialysis. Patients also pose a potential problem in that given the demographics, patients tend to be from a lower level socioeconomic background and possess a categorically under educated level than the general population. ${ }^{18}$ This lends towards a challenge for physicians even among those that have a broader vision for treatment if the patent cannot be trusted on their own for self-care. This is also a reflection of the prescribed care that places more patients in the outpatient setting.

All this does not negate the fact that two large dialysis service providers control the market with the others simply looking for the scraps of the spoils. So how did this happen? Is this a violation of antitrust? Possibly. Why would the federal government let this happen to a system that was just supposed to be a stop gap measure to ensure care for those most vulnerable in the population? In looking at the

${ }^{18}$ Sullivan JD. How end stage renal disease creates, enhances and promotes poverty for patients in the United States. Arch Renal Dis Manag. 2019;4(1):008-011. market, this is a clear violation of antitrust. Two companies should not control this amount of the market. It is unclear whether or not this impacts patient care, but it does stagnate technological advances and treatment modalities that may improve patient outcomes and also improve economic contributions to the country. The problem is that Medicare was sold a product that didn't or couldn't live up to its potential. It's true that the technology was available to treat these patients and that access to care was limited. Patients were passing away waiting for access to care and in some cases, were subjected to committees to evaluate their contributions to society to provide access to care. The government's intervention providing funding was inspirational and needed to save these patients that had a treatment but not access to care.

Altruistic arguments aside, the government has been on the payment side and not the preventative side. As a result, the number of patients that have required dialysis has skyrocketed and the government has pushed back only with cost reductions and not without patient reductions. The government's reaction has been not to get out in front of the disease, but rather to reduce expendiures via cost per treatment. While this may seem like a simple approach to getting things under control, the federal government seems to beg to differ with the seemily approach that the problem will solve itself. So, it is difficult to say whether or not the federal government looked the other way and created this oligopoly with Fresenius Medical Care and DaVita Kidney Care controlling $85 \%$ of the market of the top 10 providers. ${ }^{19}$ Normally, it would not be in the best interests of a nation to have provided this market power to a few corporations. In many cases, they would argue that is was for national safety and in the best interest of the country. This seems not to be the case in dialysis and the treatment of the disease. What seems more prevalent is a system whereby the target outcome is to reduce expenditures, but not get out ahead of the issue as to why patients are continuing to require dialysis for survival. The top two providers that control the direction of modalities through their physician network doesn't lead to preventative care or better access to other treatment options, but rather maintaining of a system that doesn't seem to be working. If the system is to work, Medicare needs to be out front and provide incentives to providers to keep patients off of dialysis. Being a catchment system just adds cost to a system that can't afford the numbers of patients with this chronic disease. After almost fifty years of operations, one conclusion seems to be prevalent. Medicare is willing to reimburse at the absolute lowest cost and that the largest providers should be broken down to incite technological advances in care.

\section{Acknowledgments}

None.

\section{Conflicts of interest}

The author declares there is no conflict of interest.

${ }^{19} \mathrm{https}$ //www.healio.com/news/nephrology/20180227/the-largest-dialysisproviders-in-2017-more-jump-o Accessed 3/20/20 